\title{
New Cells in Old Mice: The ABCs of Humoral Immunosenescence
}

\author{
Yi Hao, Michael A. Oropallo, Jean L. Scholz, and Michael P. Cancro*
}

Department of Pathology \& Laboratory Medicine, 284 John Morgan Building, 3620 Hamilton Walk, Perelman School of Medicine at the University of Pennsylvania, Philadelphia, PA 19104-6082, USA

\begin{abstract}
Dampened humoral immune responses and increased propensity for autoimmune and inflammatory diseases are hallmarks of aging. Here, we summarize recent progress in understanding how aging affects humoral immunity, focusing on the discovery of a phenotypically and functionally unique B cell subset in aged mice, its potential role in immunosenescence, and its relationship to increased inflammation and autoimmunity.
\end{abstract}

Keywords: Age, B cell homeostasis, function.

\section{OVERVIEW}

Aging is a complex process characterized by functional declines in multiple physiologic systems. Age-related alterations in the immune system, a spectrum of changes that are in toto termed "immunosenescence," yield enhanced susceptibility to infectious diseases, increased propensity for inflammatory responses and autoantibody production, and consequently, increased morbidity and mortality [1-14]. Understanding the mechanisms through which age-associated phenomena yield the overall immunosenescent phenotype presents a fundamental and challenging biological problem. Multiple factors contribute to this general deterioration of immune activity, encompassing both cell-intrinsic changes and alterations in lymphoid organ microenvironments. In particular, determining how age-associated changes result in modified homeostatic relationships among steady-state lymphocyte pools, and how this in turn impacts the initiation and quality of adaptive immune responses, may provide the keys to effective prophylaxis and intervention. In this review, we briefly summarize global shifts in humoral immune responsiveness that emerge with age, followed by a detailed consideration of changes in the genesis and homeostasis of $\mathrm{B}$ lymphocyte populations. Finally, we focus on a recently described B lineage subset that emerges with age $[15,16]$, and discuss how the shifting palette of functional B cell subsets may contribute to the global landscape of immunosenescence.

\section{AGING ALTERS HUMORAL IMMUNE RESPONSES}

Protective humoral immunity requires maintaining both naïve and antigen-experienced $\mathrm{B}$ cell subsets that respond robustly to pathogens, yet maintain self-tolerance. Multiple studies have revealed that, in contrast to healthy young adults, aged individuals display blunted humoral responses

\footnotetext{
*Address correspondence to this author at the Department of Pathology \& Laboratory Medicine, 284 John Morgan Building, 3620 Hamilton Walk, Perelman School of Medicine at the University of Pennsylvania, Philadelphia, PA 19104-6082, USA; Tel: 215-898-8067; Fax: 215-573-2350;

E-mail: cancro@mail.med.upenn.edu
}

to both new and previously encountered antigens, as well as increases in autoantibody levels [4,7,11,13,17-19]. Accordingly, studies over the last several decades have interrogated the basis for this array of features. Table I summarizes some of the changes in B cell development, dynamics and function that have been revealed by these efforts.

Early work focused primarily upon whether the frequency or clonal composition of responsive B cells changes in aged individuals, as well as whether hallmarks of effective humoral immune responses are altered. Examinations of how aging influences responding $\mathrm{B}$ cell frequencies, using model antigens in mice, have yielded mixed results. For example, aged mice have decreased frequencies of B cells responsive to some antigens, such as the haptens 2,4-dinitrophenyl (DNP) [20] and (4-hydroxy-3-nitrophenyl)acetyl (NP) [21]. In contrast, the frequencies of $\mathrm{B}$ cells for other antigens are either unchanged, or, in some cases, increased with age [2224]. More detailed analyses of clonal composition have led to the notion that while some specificities are maintained within the aged $\mathrm{B}$ cell repertoire, the relative representation of particular IgVH gene families shifts, and the responsive repertoire is relatively reduced in overall clonotypic diversity. Moreover, these shifts have been implicated in qualitative changes to antibodies produced against newly encountered antigens [25-27]. These changes in repertoire diversity and composition reflect multiple underlying mechanisms. For example, truncation of the responsive repertoire is partly explained by so-called B cell clonal expansions that emerge at the expense of more diverse naïve $B$ cell populations $[28,29]$. Furthermore, shifts in key features of developing B cells and their production rates (discussed below) may yield skewed generation or selection of naïve B cell clonotypes [30-32].

In addition to changes in the composition of naïve $\mathrm{B}$ cell pools per se, a substantial literature indicates that B lineageextrinsic components act in concert to yield overall dulled humoral responses. For example, both CD4+ and CD8+ T cells in aged mice display reduced responsiveness to encounters with novel Ag [33], and the effectiveness with which CD4+ $\mathrm{T}$ cells provide cognate help diminishes with age 
Table I. Age-associated Changes in B Cell Subsets and Compartments

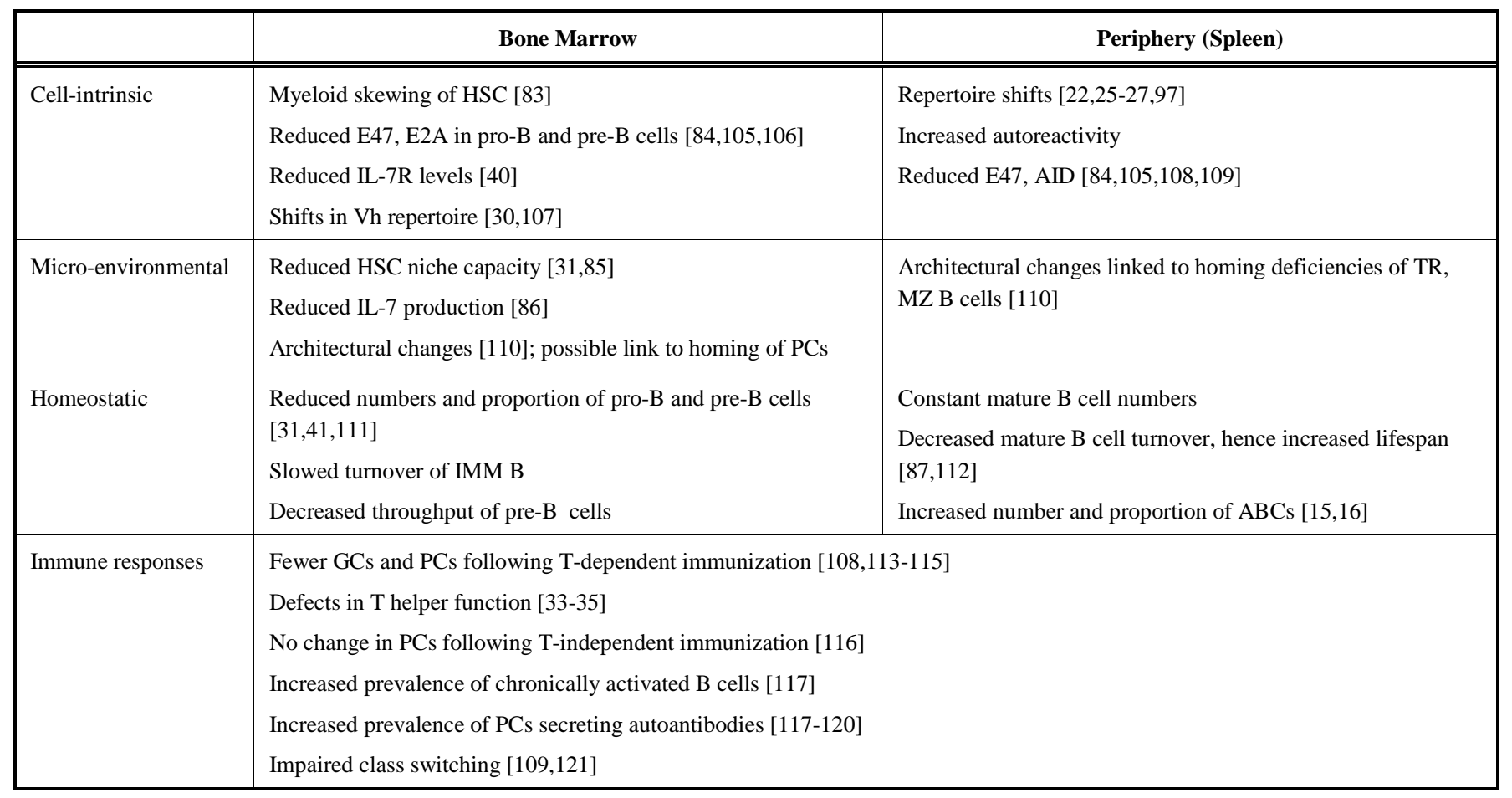

[34,35]. In addition, aging detrimentally impacts the ability of follicular dendritic cells (FDC) to participate in germinal center (GC) reactions [36,37]. Analogously, age-associated alterations in B cell functional attributes may exert trans effects on T cells via altered cytokine secretion and antigen presentation properties.

Taken together, these findings suggest that the causes of depressed humoral immunity in aging are complex, and likely involve a B cell-intrinsic loss of responsiveness and alterations in antigen receptor diversity, as well as reduced stromal and accessory cell support. With this in mind, research has focused on understanding the basis for these multifaceted defects, thus fostering detailed analyses of how developing and mature B cell populations change with age.

\section{AGING IMPACTS B LYMPHOCYTE GENERATION, SELECTION, AND HOMEOSTASIS}

Altered hematopoiesis is a consistent feature of aging, marked by increased rates of myeloid cell production and reduced rates of both $\mathrm{T}$ and $\mathrm{B}$ lymphocyte production [33, 38-42]. A brief synopsis of B cell differentiation is provided here, in order to highlight aspects that shift with age. Detailed reviews of this process are available elsewhere [4345].

In adults, $\mathrm{B}$ cells are continuously generated from bone marrow (BM) hematopoietic stem cells (HSC) that give rise to intermediates with increasingly restricted lineage alternatives. Multipotent progenitors (MPPs) derived from HSC have an equal ability to differentiate into myeloid and lymphoid lineages [45]. MPPs may differentiate into B lymphocyte-specified progenitors that, through the upregulation of key proteins and cytokine receptors, become committed to the $\mathrm{B}$ lineage as pro-B cells. The transcription factors E2A, Early B cell Factor (EBF), and Pax-5 are crucial for B lineage commitment [44]. These proteins are necessary for activating genes such as RAG and for regulating VDJ recombination, and thus $B$ cell antigen receptor (BCR) formation [46-49]. Furthermore, these transcription factors upregulate the expression of receptors requisite for $\mathrm{B}$ cell survival and subsequent differentiative cues, such as the interleukin-7 receptor (IL-7R) [50]. Successive rearrangements at the immunoglobulin (Ig) heavy and light chain loci during the proand pre-B cell stages yield differentiation into IgM+ immature (IMM) B cells [51]. IMM B cells exit the BM and migrate to the spleen to complete maturation as transitional (TR) B cells [52-57]. Following TR differentiation, these newly formed $B$ cells enter one of two mature peripheral $B$ cell pools defined by both function and anatomical location: the splenic marginal zone (MZ) or the splenic or lymph node follicle (FO).

In addition to a knowledge of these differentiative events and subsets, appreciating the influence of selection and competition in shaping $\mathrm{B}$ cell pools is fundamental to understanding the overall impact of age-related changes. Indeed, stringent selection and competition, based on both BCR specificity and homeostatic demands, occurs among emerging and mature B cells. Thus, avid BCR ligation among IMM B cells in the BM leads to receptor editing or cell death [58]. This is largely a B cell intrinsic process in which the BCR signaling thresholds for selection cannot be modified by extrinsic factors. About $90 \%$ of all IMM B cells are lost to these so-called central tolerance mechanisms. Once the surviving IMM B cells exit the BM, they continue to undergo selection based on BCR specificity during the TR stages 
[59]. During TR selection, sustained or strong BCR signals yield death or inactivation [55, 60-64]. In addition, TR B cells must also receive a minimal, or so-called "tonic", BCR signal to successfully complete differentiation [65-69]. Thus, only those TR B cells whose BCR signal strengths fall between the thresholds for these negative and positive selection processes persist. Under normal circumstances, only about $30 \%$ of TR cells survive to join the mature preimmune pools $[56,57,70]$. Within mature peripheral pools, the requisite for tonic BCR signals remains.

Because B cells are continuously produced, each subset exists at a dynamic steady state, whose magnitude is dictated by the rate at which new cells are generated and by cellular lifespan. Thus, the rate of influx from the TR pool, coupled with the longevity of FO and MZ B cells, determines steady state mature B cell numbers. B lymphocyte stimulator (BLyS, also termed BAFF), governs mature B cell homeostasis through interactions with BLyS Receptor 3 (BR3, also termed BAFFR, reviewed in [71]), which is initially expressed in the early TR stages [72]. Unlike selection at the IMM stage, the range of acceptable BCR signal strengths affording survival in the TR, FO, and MZ subsets is plastic, and can be varied by the availability of BLyS. Thus, the lack of BLyS/BR3 signaling leads to profound reductions in FO and MZ compartments [73,74] due to failed TR differentiation and shortened mature B cell lifespan [75]. Conversely, elevated BLyS levels increase the proportion of TR B cells completing maturation and extend the lifespan of $\mathrm{FO}$ and MZ B cells [72], thereby enlarging the mature pools and raising the likelihood that potentially autoreactive B cells survive TR differentiation [76].

Changes in the magnitude, kinetics, and behavior of virtually all B cell generative compartments are observed with advancing age. This reflects cell-intrinsic changes among key lymphocyte progenitors and B cell differentiation intermediates, as well as alterations in the capacity of the BM to support B cell differentiation. The numbers of hematopoietic stem cells (HSCs) remain constant or actually increase with age in mice [77,78]. However, a combination of cell-intrinsic and microenvironmental factors are thought to result in ageassociated changes in gene expression (transcription factors and cell surface proteins) [77,79], reduced expansion (selfrenewal) potential [80], reduced homing efficiency [81], engraftment potential [82], and myeloid skewed differentiation [83]. The existence of an intrinsic, genetically controlled "program" that leads to shifted HSC potential during aging has been postulated [80], but whether observed genetic changes are a cause or an effect of HSC aging is not known.

With age, the steady-state numbers of $B$ lineage differentiative intermediates change. Initial studies reported a 2- to 4-fold drop in the numbers of pre-B cells, suggesting that diminished B lymphopoiesis reflects a block at the proto pre-B cell step [41]. However, subsequent analyses have revealed that reductions are detectable in the earliest $\mathrm{B}$ lineage specified subsets [38]. Importantly, the proportional drop is greatest at the pre-B stage, suggesting that with age, there are differential effects on the molecular interactions mediating transit through successive stages of B cell development. Indeed, E47, a key component of the transcription factor
E2A, is decreased in aged pro- and pre-B cells [84]. The loss of E47 expression is likely due to post-transcriptional regulation, as mRNA levels are similar in young and old mice. RAG levels are also decreased in aged pro-B cells, resulting in a decrease in VDJ recombination and thus a decrease in successful pro- to pre-B cell differentiation [31]. Furthermore, there is an intrinsic decrease in the ability of pro- $\mathrm{B}$ cells to respond to IL-7 [40]. In sum, aging is clearly accompanied by B cell-intrinsic defects that hinder their development. The BM microenvironment also changes with age, contributing to the reduced rates of B cell genesis. For example, changes to endothelial cells reduce the capacity of the BM to create HSC-specific niches [85], and B cell progenitors are affected by decreased secretion of requisite IL-7 from BM stromal cells [86].

Despite age-associated reductions in BM output, the steady-state numbers of mature B cells remain relatively constant in mice [87]. Conflicting evidence exists as to whether circulating B cell numbers in humans similarly remain constant or instead decrease (see [88] for review). This leads to the question of how peripheral lymphocyte numbers are maintained in the face of reduced input, as well as whether the mechanisms responsible for preserving these peripheral lymphoid niches is related to blunted humoral responsiveness and autoimmunity. Indeed, multiple laboratories have demonstrated altered turnover and throughput rates in IMM, TR, and mature B cell subsets of aged individuals compared to young adults, suggesting altered homeostatic demands that might lead to shifts in selection, competition, and survival. These possibilities raise the question of whether novel B cell subsets arise with age and, if so, how they are related to previously reported changes in B cell homeostasis and function, as well as the overall immunosenescent phenotype.

\section{A PHENOTYPICALLY AND FUNCTIONALLY DIS- TINCT B CELL SUBSET ACCUMULATES WITH AGE}

A mature B cell subset that emerges with age, termed age-associated B cells (ABCs), has recently been reported by two groups. Hao et al. define ABCs as CD21/35- CD23CD19+ B cells which respond poorly to adaptive and most innate stimuli in vitro, and may directly or indirectly foster aberrant responses by secreting inflammatory cytokines and/or negative regulatory cytokines, and also by engendering Th17 differentiation [15]. Rubtsov et al. define ABCs as $\mathrm{CD} 11 \mathrm{c}+\mathrm{CD} 11 \mathrm{~b}+\mathrm{CD} 19+\mathrm{B}$ cells that may play a direct role in autoimmunity via secretion of autoantibodies [16]. Strengthening this potential relationship with autoimmunity, they found that ABCs emerge at very early ages in autoimmuneprone mice, and reported phenotypically similar cells in female rheumatoid arthritis patients [16]. A comparison of the ABCs characterized by each research team is provided in Table II. Based upon the numerous similarities, it is likely that the ABCs reported by each group are related and overlapping subsets. In both cases, ABCs accumulate with age, are more prevalent in female mice, and rely upon TLR signaling to expand. Moreover, in both cases, stimulated ABCs exhibit functional attributes associated with innate immunity, 
inflammatory responses, and/or autoimmunity. These general features are schematized in Fig. (1). In the discussion that follows, we treat ABCs as a singular subset for the sake of clarity.

The gradual emergence and persistence of this previously unappreciated, functionally novel subset raises several questions. First, what are the progenitor pools for ABCs, and what drives their generation? Second, are ABCs connected to homeostatic changes seen with advancing age, either in terms of their generation, or in terms of trans effects upon the remaining FO and MZ cells? Third, do ABCs either directly or indirectly alter the quality of immune responses, thus contributing to the increased susceptibility to infection inflammatory processes and autoantibody production that accompany aging? Finally, considering these factors in aggregate, might approaches targeting ABCs or their downstream effects help revitalize immune responses or amelio-

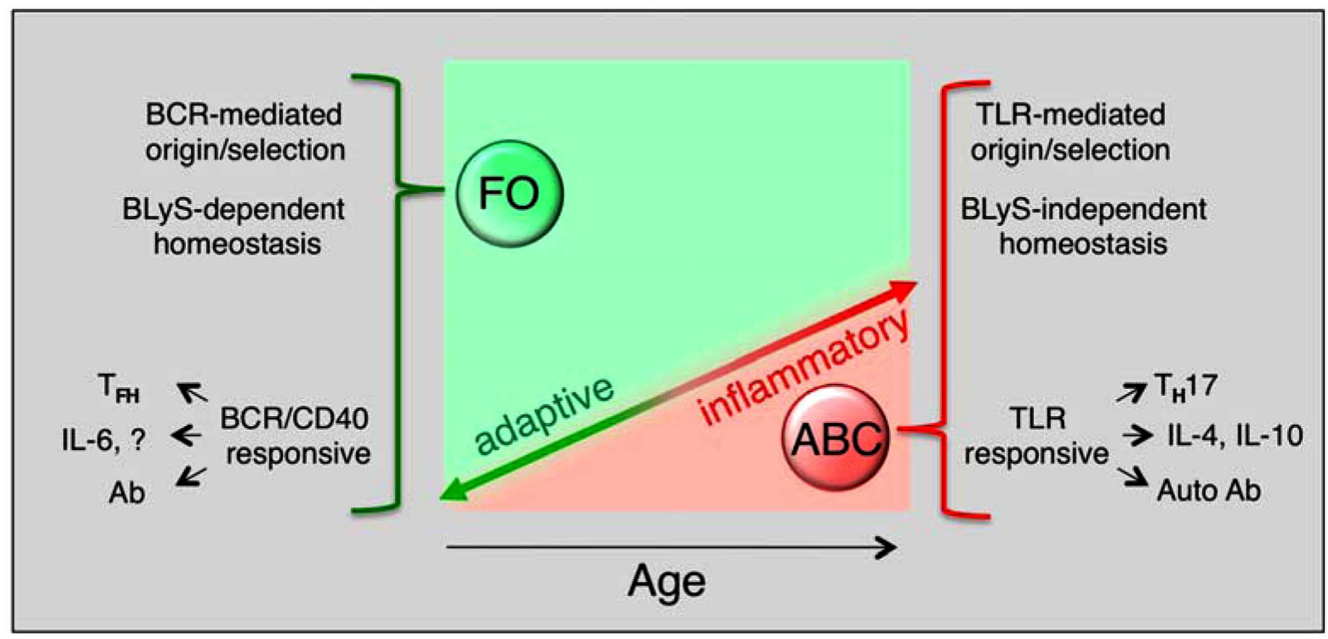

Fig. (1). Schematic of $A B C$ accumulation and impact with age. With increasing age, the proportion of ABCs (shaded red) increases, at the expense of FO and MZ subsets (shaded green). The functional attributes of ABCs differ from FO B cells. Whereas FO B cells respond to BCR crosslinking, ABCs do not, and instead respond only to TLR-driven signals. In addition, ABCs are independent of the cytokine BLyS, while FO B cells require BLyS signaling for continued survival. The effector functions of ABCs, in terms of antigen presentation, cytokine secretion, and $\mathrm{T}$ cell polarization also differ.

Table II. Properties of Age-associated B Cells

\begin{tabular}{|c|c|c|c|}
\hline & & Hao et al. [15] & Rubtsov et al. [16] \\
\hline \multirow{4}{*}{ 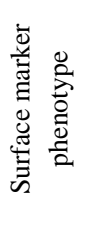 } & Defining markers & CD21- CD23- & $\mathrm{CD} 11 \mathrm{~b}+\mathrm{CD} 11 \mathrm{c}+$ \\
\hline & B lineage markers & CD19+ B220+ IgMlo IgDlo & CD19+ B220+ IgM+/- IgDlo \\
\hline & Other markers & CD11b+/- CD5- CXCR5lo CXCR4+ & CD11b+ CD5+ VCAM-1+ \\
\hline & Activation markers & MHC II+ CD86lo & MHC II+ CD86lo CD138+ \\
\hline \multirow{4}{*}{ 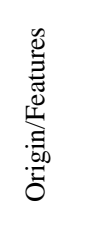 } & Gender predominance & Female $>$ male & Female $>$ male \\
\hline & Anatomic distribution & Spleen, BM, blood & Spleen, blood (BM N.D.) \\
\hline & Transcription factors & Pax5+ Blimp-1+ & Pax5+ Blimp-1+ \\
\hline & Generation/progenitors & TLR-driven/FO & TLR-driven/? \\
\hline \multirow{3}{*}{ 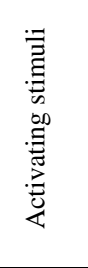 } & Anti-IgM \pm anti-CD40 & No & N.D. \\
\hline & TLR 7 & $\begin{array}{l}\text { Yes, modest } \\
\text { augmented by anti-IgM }\end{array}$ & Yes, robust \\
\hline & TLR 9 & $\begin{array}{l}\text { Yes, robust } \\
\text { augmented by anti-IgM }\end{array}$ & N.D. \\
\hline \multirow{3}{*}{ 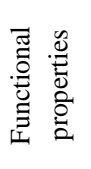 } & Autoantibody production & Undetectable anti-dsDNA & Yes, anti-chromatin \\
\hline & Cytokine production & High IL-4, IL-10 & N.D. \\
\hline & Antigen presentation & Yes, skews CD4+ T cells to Th17 & N.D. \\
\hline
\end{tabular}

+/- indicates presence of some cells that are + and some that are -

N.D.: not done 
rate age-associated disease susceptibilities in the elderly?

\section{ABCS ORIGINATE FROM MATURE B CELL PRO- GENITORS VIA TLR-MEDIATED PROCESSES}

The near-absence of cells with the ABC-defining phenotype in young adult mice raises the question of how ABCs arise. Autoreconstitution studies indicate that ABCs are not simply the product of $B$ cell generation in aged BM, because their frequency and number in sublethally irradiated aged mice are extremely low after 12 weeks, despite full reconstitution of all other developmental and primary B cell subsets [15]. Instead, there is strong evidence that Toll-like receptor (TLR) signaling plays a critical role in the generation of ABCs from pre-existing mature $\mathrm{B}$ cell progenitors. Both aged and young FO B cells acquire the ABC phenotype upon TLR9 stimulation in vitro [15]. Moreover, TLR7 and MyD88 signaling are required for ABC development, as aged TLR7 or MyD88 knockout mice fail to accumulate ABCs [16]. Finally, 30 days after adoptive transfer into replete hosts, a very small proportion of the donor FO B cells acquire characteristics of ABCs after extensive division. Thus, FO B cells are the likely progenitors of ABCs, though MZ and/or TR progenitors cannot be ruled out; and chronic TLR signaling is apparently required to drive expansion and differentiation to the $\mathrm{ABC}$ phenotype. It remains to be seen whether all FO B cells have the potential to differentiate into ABCs, such that stochastic mechanisms limit the numbers that acquire full ABC character; or if instead, ABCs are derived from a particular subset of clones within the FO pool. Accordingly, determining whether ABCs display clonotypic bias congruent with $\mathrm{B}$ cell clonal expansions might prove informative in terms of both their origin and driving forces. For example, MZ and B1 subsets display clear clonotypic biases that might be evident if they serve as $\mathrm{ABC}$ progenitors [89-92]. Alternatively, only B cells with BCRs that bind antigens associated with TLR7 and TLR9 ligands might be responsible for the accumulation of ABCs, regardless of subset. These possibilities are not mutually exclusive, and should prove approachable with current technologies that allow detailed repertoire and specificity analyses in aged B cell pools [29].

\section{THE INTERACTION OF ABCs WITH BLyS: IMPLI- CATIONS FOR B CELL HOMEOSTASIS}

Regardless of exact origin, determining how the continuously enlarging ABC subset impacts homeostasis of other B cell subsets is key to understanding their overall impact. Given the central role of BLyS in determining the set point for primary B cell numbers, it is striking that despite similar BLyS receptor levels and BLyS binding capacity, ABCs do not depend upon BLyS for survival. This is evidenced by their robust survival and lack of BLyS dependence compared to $\mathrm{FO}$ and $\mathrm{MZ} \mathrm{B}$ cells in vitro, as well as their persistence following in vivo BLyS blockade [15]. Thus, the molecular basis for BLyS-independent ABC survival, as well as how their consumption of BLyS impacts the selection and dynamics of BLyS dependent pre-immune pools, demands further exploration.
BLyS signaling through BR3 provides survival signals through non-canonical NF- $\mathrm{B}$ activation and "crosstalk" with multiple downstream elements of tonic BCR signaling [93-95], yielding survival through upregulation of antiapoptotic members of the Bcl-2 family [72] and metabolic mediators [96]. Thus the BLyS independence of ABCs may reflect a myriad of factors, including altered BR3 or BCR signals, compensatory signaling and crosstalk from TLRs, or other, as yet unknown stimuli that mimic these pathways. One clue to this puzzle may be the observation that BCR ligation acts synergistically with TLR9 signals in ABC activation, whereas BCR stimulation alone has no effect on ABCs [15]. This implies that BCR signaling is fundamentally different in ABCs, inasmuch as it can deliver or modify signals in the context of TLR driven downstream elements, but mediates neither the proliferation- nor death-inducing events associated with BCR ligation in FO and MZ cells.

Apart from the molecular mechanisms underlying BLyS independence, this physiological property implies that a profound shift in B cell homeostasis occurs with advancing age: the mature $\mathrm{B}$ cell niche is increasingly populated by cells that do not depend on BLyS for survival yet consume BLyS effectively - thus limiting BLyS availability to other mature subsets. The question of whether ABCs show exceptional cellular lifespan, as well as whether they act as "supercompetitors" in the pre-immune B cell niche, remains to be addressed. Indeed, slowed turnover of the overall mature, pre-immune B cell pool has been well-established through in vivo labeling studies [87]. Inasmuch as these prior studies analyzed mature splenic B cells in aggregate, it is tempting to speculate that ABCs may contribute disproportionately to these protracted kinetics. Alternatively, because ABCs will increasingly usurp available BLyS, both FO and MZ B cells may themselves be more stringently selected. This would yield a situation in which only the strongest homeostatic competitors, which are longer lived, would remain in the FO and MZ pools. Indeed, the observation that FO B cells from aged mice show improved survival in vitro compared to those from young mice is consistent with the notion that only the "most fit" FO B cells persist in the presence of ABCs.

An increased incidence of B-cell clonal expansions accompanies age in both mice and humans. This has led to the suggestion that genetic changes coupled with selection processes underlie the "evolution" of persistent B-cell clonal expansions $[28,97]$. The establishment of ABCs from chronically stimulated and expanded FO and MZ progenitors would be consistent with this hypothesis, as such alterations might free ABCs from BLyS-tuned selection processes.

\section{DO ABCS INFLUENCE IMMUNE RESPONSIVENESS AND TOLERANCE?}

The growing presence of ABCs, coupled with their novel functional attributes, prompts consideration of their contributions to the features of immunosenescence. In general, ABCs could contribute to age-associated declines in humoral responsiveness and propensity for chronic inflammation or autoantibody production either directly, based on their distinct activation requisites; or through trans effects mediated 
by their proinflammatory and regulatory cytokine production and antigen presentation capacities. There is suggestive evidence for each of these possibilities.

Unlike FO B cells, which respond to BCR ligation and costimulation in vitro, ABCs instead respond to TLR9 and/or TLR7 stimuli, particularly in combination with BCR ligation $[15,16]$. This resistance to classically T-dependent modes of activation, coupled with a reliance on TLR stimulation, might limit the quality of $\mathrm{ABC}$ responses to the short-lived extrafollicular effectors characteristic of T-independent antigens. Moreover, TLRs are commonly associated with skewing towards inflammatory responses [98]. Thus, steadily increasing numbers of ABCs may yield more prominent innate-like immune responses, characterized by low affinity antibody and inflammatory processes, instead of GC initiation, affinity maturation, and memory B cell differentiation.

In addition to such direct effects on humoral responses, ABCs likely exert trans effects through their secretion of regulatory and inflammatory cytokines, as well as their antigen presentation abilities. Following in vitro stimulation, ABCs produce regulatory cytokines, such as IL-10 [15], that have been shown to inhibit adaptive immunity [99]. Moreover, they also secrete inflammatory cytokines that can further alter response quality. Interestingly, $A B C s$ preferentially skew activated CD4+ $\mathrm{T}$ cells to a Th17 fate compared to presentation mediated by young FO B cells, aged FO B cells, or dendritic cells [15]. Thus, during initial antigen encounter, ABCs may foster Th17 differentiation at the expense of $\mathrm{T}$ follicular helper generation, again thwarting GC formation, affinity maturation, and memory cell formation.

These properties are equally relevant to the potential role for ABCs in autoimmunity. Thus, TLR7 and TLR9 are wellestablished factors in autoantibody secretion and autoimmune disease development [100,101]. Indeed, following TLR7 activation in vitro, ABCs produce anti-chromatin antibody, directly implicating ABCs in the increased autoantibody titers observed in aged mice [16]. Similarly, Th17 cells secrete a range of cytokines that exacerbate inflammatory/autoimmune diseases [102,103]. Thus, ABC-mediated antigen presentation may contribute to a broader susceptibility background in aged individuals that includes elevated proinflammatory cytokines and shifts in $\mathrm{T}$ cell-associated immune responses $[14,18,19,102,104]$. Together, these attributes suggest that frank humoral autoimmune disease, which often emerges in young or middle aged adults, might be associated with premature accretion of ABC. The early accumulation of ABCs observed by Rubtsov et al. in autoimmune prone mice, as well as in middle-aged RA and scleroderma patients, is consistent with this possibility[16].

\section{ARE ABCs TARGETS FOR INTERVENTION?}

It is tempting to speculate that approaches either targeting $\mathrm{ABCs}$ per se, or leveraging their functional characteristics to advantage, might be useful in either rejuvenating immune responses or changing the course of inflammatory or autoimmune diseases. For example, vaccine efficacy might be improved through the design of antigen/adjuvant systems that stimulate both the TLR and BCR receptors of ABCs. If ABCs promote Th17 responses in aged individuals, then ablating ABCs might help to reduce the likelihood or progression of inflammatory/autoimmune disorders. While these are tantalizing conjectures, a deeper understanding of the origins, attributes, and functional consequences of ABCs is clearly required before specific targets can be identified or evaluated.

\section{SUMMARY}

B cell generation, subset composition, diversity, and function are altered with age. A previously unappreciated mature B cell subset that accumulates with age has recently been identified. Cells of this age-associated B cell (ABC) subset are phenotypically distinct from other mature subsets, and appear to favor innate, inflammatory immune responses. The accumulation of the ABC subset may bias the immune system toward increased propensity for inflammatory or autoimmune responses, and/or impaired "classical" responses that generate robust plasma cell production and memory. Important aspects of the $\mathrm{ABC}$ subset that await further investigation include the identification of progenitors, requisites for persistence, and possible alterations in BCR- and TLRmediated signaling, as well as a determination of their precise role in autoimmunity. Finally, while it is tempting to speculate that ABC-targeted approaches might improve some aspects of immunosenescence, further understanding of their origins and functional attributes is required before such possibilities can be critically evaluated.

\section{ACKNOWLEDGEMENTS}

This work was supported by U.S.P.H.S. grants AG030227 and AI073939 to MPC. The authors thank Laurie Baker for expert editorial assistance.

\section{CONFLICT OF INTEREST}

None declared.

\section{REFERENCES}

[1] Pawelec G, Solana R. Immunosenescence. Immunol Today 1997; 18: 514-6.

[2] Franceschi C, Passeri M, De Benedictis G, Motta L. Immunosenescence . Aging (Milano) 1998; 10: 153-4.

[3] Franceschi C, Bonafe M, Valensin S. Human immunosenescence: the prevailing of innate immunity, the failing of clonotypic immunity, and the filling of immunological space. Vaccine 2000; 18: 1717-20.

[4] Ginaldi L, Loreto MF, Corsi MP, Modesti M, De Martinis M. Immunosenescence and infectious diseases. Microbes Infect 2001; 3: 851-7.

[5] Malaguarnera L, Ferlito L, Imbesi RM, et al. Immunosenescence: a review. Arch Gerontol Geriatr 2001; 32: 1-14.

[6] Tarazona R, Solana R, Ouyang Q, Pawelec G. Basic biology and clinical impact of immunosenescence. Exp Gerontol 2002; 37: 1839.

[7] Pawelec G. Immunosenescence and vaccination. Immun Ageing 2005; 2: 16.

[8] Aw D, Silva AB, Palmer DB. Immunosenescence: emerging challenges for an ageing population. Immunology 2007; 120: 435-46.

[9] Hakim FT, Gress RE. Immunosenescence: deficits in adaptive immunity in the elderly. Tissue Antigens 2007; 70: 179-89.

[10] Thewissen M, Stinissen P. New concepts on the pathogenesis of autoimmune diseases: a role for immune homeostasis, immunoregulation, and immunosenescence. Crit Rev Immunol 2008; 28: 363-76. 
[11] Grubeck-Loebenstein B, Della Bella S, Iorio AM, Michel JP, Pawelec G, Solana R. Immunosenescence and vaccine failure in the elderly. Aging Clin Exp Res 2009; 21: 201-9.

[12] Ongradi J, Stercz B, Kovesdi V, Vertes L. Immunosenescence and vaccination of the elderly, I. Age-related immune impairment. Acta Microbiol Immunol Hung 2009; 56: 199-210.

[13] Sambhara S, McElhaney JE. Immunosenescence and influenza vaccine efficacy. Curr Top Microbiol Immunol 2009; 333: 413-29.

[14] Lindstrom TM, Robinson WH. Rheumatoid arthritis: a role for immunosenescence? J Am Geriatr Soc 2010; 58: 1565-75.

[15] Hao Y, O'Neill PJ, Naradikian MS, Scholz JL, Cancro MP. A Bcell subset uniquely responsive to innate stimuli accumulates in aged mice. Blood 2011; 118 (5): 1294-304

[16] Rubtsov AV, Rubtsova K, Fischer A, et al. TLR7-driven accumulation of a novel CD11c+ B-cell population is important for the development of autoimmunity. Blood 2011; 118 (5): 1305-15.

[17] Stacy S, Krolick KA, Infante AJ, Kraig E. Immunological memory and late onset autoimmunity. Mech Ageing Dev 2002; 123: 975-85.

[18] Boren E, Gershwin ME. Inflamm-aging: autoimmunity, and the immune-risk phenotype. Autoimmun Rev 2004; 3: 401-6.

[19] Grolleau-Julius A, Ray D, Yung RL. The role of epigenetics in aging and autoimmunity. Clin Rev Allergy Immunol 2010; 39: 4250.

[20] Zharhary D, Klinman NR. Antigen responsiveness of the mature and generative B cell populations of aged mice. J Exp Med 1983; 157: $1300-8$

[21] Zharhary D, Klinman NR. The frequency and fine specificity of B cells responsive to (4-hydroxy-3-nitrophenyl)acetyl in aged mice. Cell Immunol 1986; 100: 452-61.

[22] Nicoletti C, Cerny J. The repertoire diversity and magnitude of antibody responses to bacterial antigens in aged mice: I. Ageassociated changes in antibody responses differ according to the mouse strain. Cell Immunol 1991; 133: 72-83.

[23] Zharhary D, Klinman NR. B cell repertoire diversity to PR8 influenza virus does not decrease with age. J Immunol 1984; 133: 22857.

[24] Zharhary D, Klinman NR. A selective increase in the generation of phosphorylcholine-specific B cells associated with aging. J Immunol 1986; 136: 368-70.

[25] Riley SC, Froscher BG, Linton PJ, Zharhary D, Marcu K, Klinman $\mathrm{NR}$. Altered $\mathrm{VH}$ gene segment utilization in the response to phosphorylcholine by aged mice. J Immunol 1989; 143: 3798-805.

[26] Nicoletti C, Borghesi-Nicoletti C, Yang XH, Schulze DH, Cerny J. Repertoire diversity of antibody response to bacterial antigens in aged mice. II. Phosphorylcholine-antibody in young and aged mice differ in both VH/VL gene repertoire and in specificity. J Immunol 1991; 147: 2750-5.

[27] Nicoletti C, Yang X, Cerny J. Repertoire diversity of antibody response to bacterial antigens in aged mice. III. Phosphorylcholine antibody from young and aged mice differ in structure and protective activity against infection with Streptococcus pneumoniae. J Immunol 1993; 150: 543-9.

[28] Szabo P, Li F, Mathew J, Lillvis J, Weksler ME. Evolution of Bcell clonal expansions with age. Cell Immunol 2004; 231: 158-67.

[29] Gibson KL, Wu YC, Barnett Y, et al. B-cell diversity decreases in old age and is correlated with poor health status. Aging Cell 2009; 8: 18-25.

[30] Szabo P, Shen S, Telford W, Weksler ME. Impaired rearrangement of IgH V to DJ segments in bone marrow Pro-B cells from old mice. Cell Immunol 2003; 222: 78-87.

[31] Labrie JE 3rd, Sah AP, Allman DM, Cancro MP, Gerstein RM. Bone marrow microenvironmental changes underlie reduced RAGmediated recombination and B cell generation in aged mice. J Exp Med 2004; 200: 411-23.

[32] Alter-Wolf S, Blomberg BB, Riley RL. Deviation of the B cell pathway in senescent mice is associated with reduced surrogate light chain expression and altered immature B cell generation, phenotype, and light chain expression. J Immunol 2009; 182: 138-47.

[33] Maue AC, Yager EJ, Swain SL, Woodland DL, Blackman MA, Haynes L. T-cell immunosenescence: lessons learned from mouse models of aging. Trends Immunol 2009; 30: 301-5.

[34] Eaton SM, Burns EM, Kusser K, Randall TD, Haynes L. Agerelated defects in CD4 T cell cognate helper function lead to reductions in humoral responses. J Exp Med 2004; 200: 1613-22.
[35]

[50] Fleming HE, Paige CJ. Cooperation between IL-7 and the pre-B cell receptor: a key to B cell selection. Semin Immunol 2002; 14 423-30.

[51] Monroe JG, Dorshkind K. Fate decisions regulating bone marrow and peripheral B lymphocyte development. Adv Immunol 2007; 95: $1-50$

[52] Carsetti R, Rosado MM, Wardmann H. Peripheral development of B cells in mouse and man. Immunol Rev 2004; 197: 179-91.

[53] Cancro MP. Peripheral B-cell maturation: the intersection of selection and homeostasis. Immunol Rev 2004; 197: 89-101.

[54] Allman D, Lindsley RC, DeMuth W, Rudd K, Shinton SA, Hardy RR. Resolution of three nonproliferative immature splenic B cell subsets reveals multiple selection points during peripheral B cell maturation. J Immunol 2001; 167: 6834-40.

[55] Carsetti R, Kohler G, Lamers MC. Transitional B cells are the target of negative selection in the B cell compartment. J Exp Med 1995; 181: 2129-40.

[56] Allman DM, Ferguson SE, Lentz VM, Cancro MP. Peripheral B cell maturation. II. Heat-stable antigen(hi) splenic B cells are an immature developmental intermediate in the production of longlived marrow-derived B cells. J Immunol 1993; 151: 4431-44.

[57] Allman DM, Ferguson SE, Cancro MP. Peripheral B cell maturation. I. Immature peripheral B cells in adults are heat-stable antigenhi and exhibit unique signaling characteristics. J Immunol 1992; 149: 2533-40.

[58] Hartley SB, Crosbie J, Brink R, Kantor AB, Basten A, Goodnow CC. Elimination from peripheral lymphoid tissues of self-reactive B lymphocytes recognizing membrane-bound antigens. Nature 1991; 353: 765-9.

[59] Goodnow CC, Crosbie J, Adelstein S, et al. Altered immunoglobulin expression and functional silencing of self-reactive B lymphocytes in transgenic mice. Nature 1988; 334: 676-82. 
[60] Chen C, Nagy Z, Prak EL, Weigert M. Immunoglobulin heavy chain gene replacement: a mechanism of receptor editing. Immunity 1995; 3: 747-55.

[61] Nemazee DA, Burki K. Clonal deletion of B lymphocytes in a transgenic mouse bearing anti-MHC class I antibody genes. Nature 1989; 337: 562-6.

[62] Radic MZ, Erikson J, Litwin S, Weigert M. B lymphocytes may escape tolerance by revising their antigen receptors. J Exp Med 1993; 177: 1165-73.

[63] Fulcher DA, Basten A. Reduced life span of anergic self-reactive B cells in a double-transgenic model. J Exp Med 1994; 179: 125-34.

[64] Sandel PC, Monroe JG. Negative selection of immature B cells by receptor editing or deletion is determined by site of antigen encounter. Immunity 1999; 10: 289-99.

[65] Lam KP, Kuhn R, Rajewsky K. In vivo ablation of surface immunoglobulin on mature $\mathrm{B}$ cells by inducible gene targeting results in rapid cell death. Cell 1997; 90: 1073-83.

[66] Bannish G, Fuentes-Panana EM, Cambier JC, Pear WS, Monroe JG. Ligand-independent signaling functions for the B lymphocyte antigen receptor and their role in positive selection during $\mathrm{B}$ lymphopoiesis. J Exp Med 2001; 194: 1583-96.

[67] Freitas AA, Lembezat MP, Coutinho A. Expression of antibody Vregions is genetically and developmentally controlled and modulated by the B lymphocyte environment. Int Immunol 1989; 1: 34254.

[68] Gu H, Tarlinton D, Muller W, Rajewsky K, Forster I. Most peripheral B cells in mice are ligand selected. J Exp Med 1991; 173: 1357-71.

[69] Levine MH, Haberman AM, Sant'Angelo DB, et al. A B-cell receptor-specific selection step governs immature to mature B cell differentiation. Proc Natl Acad Sci USA 2000; 97: 2743-8.

[70] Osmond DG. Proliferation kinetics and the lifespan of B cells in central and peripheral lymphoid organs. Curr Opin Immunol 1991; 3: 179-85.

[71] Miller JP, Stadanlick JE, Cancro MP. Space, selection, and surveillance: setting boundaries with BLyS. J Immunol 2006; 176: 640510.

[72] Hsu BL, Harless SM, Lindsley RC, Hilbert DM, Cancro MP. Cutting edge: BLyS enables survival of transitional and mature B cells through distinct mediators. J Immunol 2002; 168: 5993-6.

[73] Schiemann B, Gommerman JL, Vora K, et al. An essential role for BAFF in the normal development of B cells through a BCMAindependent pathway. Science 2001; 293: 2111-4.

[74] Miller DJ, Hayes CE. Phenotypic and genetic characterization of a unique B lymphocyte deficiency in strain A/WySnJ mice. Eur J Immunol 1991; 21: 1123-30.

[75] Harless SM, Lentz VM, Sah AP, et al. Competition for BLySmediated signaling through Bcmd/BR3 regulates peripheral B lymphocyte numbers. Curr Biol 2001; 11: 1986-9.

[76] Thien M, Phan TG, Gardam S, et al. Excess BAFF rescues selfreactive $B$ cells from peripheral deletion and allows them to enter forbidden follicular and marginal zone niches. Immunity 2004; 20: 785-98.

[77] Rossi DJ, Bryder D, Zahn JM, et al. Cell intrinsic alterations underlie hematopoietic stem cell aging. Proc Natl Acad Sci USA 2005; 102: 9194-9.

[78] Waterstrat A, Van Zant G. Effects of aging on hematopoietic stem and progenitor cells. Curr Opin Immunol 2009; 21: 408-13.

[79] Chambers SM, Shaw CA, Gatza C, Fisk CJ, Donehower LA, Goodell MA. Aging hematopoietic stem cells decline in function and exhibit epigenetic dysregulation. PLoS Biol 2007; 5: e201.

[80] Kamminga LM, van Os R, Ausema A, et al. Impaired hematopoietic stem cell functioning after serial transplantation and during normal aging. Stem Cells 2005; 23: 82-92.

[81] Liang Y, Van Zant G, Szilvassy SJ. Effects of aging on the homing and engraftment of murine hematopoietic stem and progenitor cells. Blood 2005; 106: 1479-87.

[82] Yilmaz OH, Kiel MJ, Morrison SJ. SLAM family markers are conserved among hematopoietic stem cells from old and reconstituted mice and markedly increase their purity. Blood 2006; 107: 924-30.

[83] Dykstra B, de Haan G. Hematopoietic stem cell aging and selfrenewal. Cell Tissue Res 2008; 331: 91-101.
[84] Frasca D, Van Der Put E, Riley RL, Blomberg BB. Age-related differences in the E2A-encoded transcription factor E47 in bone marrow-derived B cell precursors and in splenic B cells. Exp Gerontol 2004; 39: 481-9.

[85] Ju Z, Jiang H, Jaworski M, et al. Telomere dysfunction induces environmental alterations limiting hematopoietic stem cell function and engraftment. Nat Med 2007; 13: 742-7.

[86] Stephan RP, Reilly CR, Witte PL. Impaired ability of bone marrow stromal cells to support B-lymphopoiesis with age. Blood 1998; 91: 75-88.

[87] Kline GH, Hayden TA, Klinman NR. B cell maintenance in aged mice reflects both increased B cell longevity and decreased B cell generation. J Immunol 1999; 162: 3342-9.

[88] Ademokun A, Wu YC, Dunn-Walters D. The ageing B cell population: composition and function. Biogerontology 2010; 11: 125-37.

[89] Martin F, Kearney JF. Marginal-zone B cells. Nat Rev Immunol 2002; 2: 323-35.

[90] Lopes-Carvalho T, Kearney JF. Development and selection of marginal zone B cells. Immunol Rev 2004; 197: 192-205.

[91] Wang H, Clarke SH. Positive selection focuses the VH12 B-cell repertoire towards a single B1 specificity with survival function. Immunol Rev 2004; 197: 51-9.

[92] Herzenberg LA, Baumgarth N, Wilshire JA. B-1 cell origins and VH repertoire determination. Curr Top Microbiol Immunol 2000; 252: 3-13.

[93] Stadanlick JE, Cancro MP. Unraveling the warp and weft of B cell fate. Immunity 2006; 25: 395-6.

[94] Stadanlick JE, Kaileh M, Karnell FG, et al. Tonic B cell antigen receptor signals supply an NF-kappaB substrate for prosurvival BLyS signaling. Nat Immunol 2008; 9: 1379-87.

[95] Otipoby KL, Sasaki Y, Schmidt-Supprian M, et al. BAFF activates Akt and Erk through BAFF-R in an IKK1-dependent manner in primary mouse B cells. Proc Natl Acad Sci USA 2008; 105: 124358.

[96] Woodland RT, Fox CJ, Schmidt MR, et al. Multiple signaling pathways promote B lymphocyte stimulator dependent B-cell growth and survival. Blood 2008; 111: 750-60.

[97] Weksler ME. Changes in the B-cell repertoire with age. Vaccine 2000; 18: 1624-8.

[98] Kawai T, Akira S. The role of pattern-recognition receptors in innate immunity: update on Toll-like receptors. Nat Immunol 2010; 11: 373-84.

[99] Ouyang W, Rutz S, Crellin NK, Valdez PA, Hymowitz SG. Regulation and functions of the IL-10 family of cytokines in inflammation and disease. Annu Rev Immunol 2010; 29: 71-109.

[100] Christensen SR, Shupe J, Nickerson K, Kashgarian M, Flavell RA, Shlomchik MJ. Toll-like receptor 7 and TLR9 dictate autoantibody specificity and have opposing inflammatory and regulatory roles in a murine model of lupus. Immunity 2006; 25: 417-28.

[101] Nickerson KM, Christensen SR, Shupe J, et al. TLR9 regulates TLR7- and MyD88-dependent autoantibody production and disease in a murine model of lupus. J Immunol 2010; 184: 1840-8.

[102] Ouyang X, Yang Z, Zhang R, et al. Potentiation of Th17 cytokines in aging process contributes to the development of colitis. Cell Immunol 2011; 266: 208-17.

[103] Yamada H. Current perspectives on the role of IL-17 in autoimmune disease. J Inflamm Res 2010; 3: 33-44.

[104] Krabbe KS, Pedersen M, Bruunsgaard H. Inflammatory mediators in the elderly. Exp Gerontol 2004; 39: 687-99.

[105] Frasca D, Nguyen D, Riley RL, Blomberg BB. Decreased E12 and/or E47 transcription factor activity in the bone marrow as well as in the spleen of aged mice. J Immunol 2003; 170: 719-26.

[106] Riley RL, Van der Put E, King AM, Frasca D, Blomberg BB. Deficient $\mathrm{B}$ lymphopoiesis in murine senescence: potential roles for dysregulation of E2A, Pax-5, and STAT5. Semin Immunol 2005; 17: 330-6.

[107] Guerrettaz LM, Johnson SA, Cambier JC. Acquired hematopoietic stem cell defects determine B-cell repertoire changes associated with aging. Proc Natl Acad Sci USA 2008; 105: 11898-902.

[108] Frasca D, Blomberg BB. Effects of aging on B cell function. Curr Opin Immunol 2009; 21: 425-30.

[109] Frasca D, Van der Put E, Riley RL, Blomberg BB. Reduced Ig class switch in aged mice correlates with decreased E47 and activation-induced cytidine deaminase. J Immunol 2004; 172: 2155-62. 
[110] Wols HA, Johnson KM, Ippolito JA, et al. Migration of immature and mature $\mathrm{B}$ cells in the aged microenvironment. Immunology 2010; 129: 278-90.

[111] Riley RL, Kruger MG, Elia J. B cell precursors are decreased in senescent BALB/c mice, but retain normal mitotic activity in vivo and in vitro. Clin Immunol Immunopathol 1991; 59: 301-13.

[112] Johnson KM, Owen K, Witte PL. Aging and developmental transitions in the B cell lineage. Int Immunol 2002; 14: 1313-23.

[113] Goidl EA, Innes JB, Weksler ME. Immunological studies of aging. II. Loss of IgG and high avidity plaque-forming cells and increased suppressor cell activity in aging mice. J Exp Med 1976; 144: 103748.

[114] Han S, Yang K, Ozen Z, et al. Enhanced differentiation of splenic plasma cells but diminished long-lived high-affinity bone marrow plasma cells in aged mice. J Immunol 2003; 170: 1267-73.

[115] Zheng B, Han S, Takahashi Y, Kelsoe G. Immunosenescence and germinal center reaction. Immunol Rev 1997; 160: 63-77.
[116] Zharhary D, Segev Y, Gershon H. The affinity and spectrum of cross reactivity of antibody production in senescent mice: the IgM response. Mech Ageing Dev 1977; 6: 385-92.

[117] Johnson SA, Rozzo SJ, Cambier JC. Aging-dependent exclusion of antigen-inexperienced cells from the peripheral B cell repertoire. J Immunol 2002; 168: 5014-23.

[118] Weksler ME, Schwab R, Huetz F, Kim YT, Coutinho A. Cellular basis for the age-associated increase in autoimmune reactions. Int Immunol 1990; 2: 329-35.

[119] Rowley MJ, Buchanan H, Mackay IR. Reciprocal change with age in antibody to extrinsic and intrinsic antigens. Lancet 1968; 2: 24-6.

[120] Eaton-Bassiri AS, Mandik-Nayak L, Seo SJ, Madaio MP, Cancro MP, Erikson J. Alterations in splenic architecture and the localization of anti-double-stranded DNA B cells in aged mice. Int Immunol 2000; 12: 915-26.

[121] Frasca D, Riley RL, Blomberg BB. Humoral immune response and B-cell functions including immunoglobulin class switch are downregulated in aged mice and humans. Semin Immunol 2005; 17:

378-84.

Received: July 11, 2011

Revised: September 14, 2011

Accepted: November 18, 2011

(C) Hao et al.; Licensee Bentham Open.

This is an open access article licensed under the terms of the Creative Commons Attribution Non-Commercial License (http://creativecommons.org/licenses/by-nc/3.0/) which permits unrestricted, non-commercial use, distribution and reproduction in any medium, provided the work is properly cited. 